\title{
Treatment of Seborrheic Keratosis by High Frequency Focused Ultrasound - An Early Experience with II Consecutive Cases
}

\author{
Jacek Calik', Monika Migdal', Tomasz Zawada $\mathbb{D}^{2}$, Torsten Bove $\mathbb{D}^{2}$ \\ 'Old Town Clinic, Wrocław, Poland; ${ }^{2}$ TOOsonix A/S, Hoersholm, Denmark \\ Correspondence: Torsten Bove, TOOsonix A/S, Agern Alle I, Hoersholm, DK-2970, Denmark, Tel +45- 2059 2999, Email torsten.bove@toosonix.com
}

\begin{abstract}
Purpose: High intensity focused ultrasound operating at $20 \mathrm{MHz}$ has been demonstrated as a safe and efficient treatment modality for a range of dermatological indications. The method is potentially also applicable to removal of seborrheic keratosis.

Patients and Methods: A total of 54 seborrheic keratoses in 11 volunteer subjects ( 8 women and 3 men, average age $51.5 \pm 13.2$ years) were treated in a single session with a medical $20 \mathrm{MHz}$ high intensity focused ultrasound device developed for dermatological conditions. Handpieces with nominal focal depths of $0.8 \mathrm{~mm}$ below the skin surface were used to administer acoustic energy of $0.99-$ $1.2 \mathrm{~J} /$ dose. An integrated dermoscope in the handpiece was used to monitor the treatment in real-time. Treatment efficacy and sideeffects were assessed directly after treatment and at follow-up 4-15 weeks after treatment.

Results: The treatment showed positive results in $96.3 \%$ of the cases. About $68.5 \%$ of the cases were classified as complete response and $27.8 \%$ of the cases as partial response. Two cases $(3.7 \%)$ did not respond to treatment and were classified as stable condition. No subjects experienced worsening of their condition, and no treatment received the classification of progressive condition. Side effects were primarily redness in the treatment area due to superficial telangiectasia, mild scarring, and persisting and slow-healing lichen planus-like keratosis. No adverse events were observed.

Conclusion: HIFU is concluded to be a safe and efficient skin treatment for seborrheic keratoses. It has advantages over conventional treatments that can lead to pain during treatment and scarring after healing.
\end{abstract}

Keywords: seborrheic warts, solar lentigo, skin tumor, HIFU, dermoscopy, dermatology

\section{Introduction}

Over the last decade, non-invasive high intensity focused ultrasound (HIFU) has been established as a safe and efficient treatment modality for critical internal cancers of major organs, ${ }^{1-5}$ bone metastases ${ }^{6}$ and cerebral pathologies. ${ }^{7}$ Direct ablative treatments are performed by positioning focal zones deep within the body, where a combination of localized heating to temperatures of about $65^{\circ} \mathrm{C}$, combined with mechanical effects from cavitation, destroy targeted cell volumes without affecting adjacent structures. ${ }^{1}$ More recently, preclinical studies using non-ablative HIFU as a means of accelerating selective drug uptake and activating certain immunological responses have furthermore shown very promising results. ${ }^{8}$ The clinical results and high research activity in new areas therefore point to a broader acceptance of HIFU as a general therapeutic tool in the future.

In the above-mentioned medical treatment modalities, the HIFU systems typically operate at frequencies from $500 \mathrm{kHz}$ to approximately $3 \mathrm{MHz}$. The operation frequency of a HIFU system is always a compromise between the needed penetration depth and the size/resolution of the focal zone in the target. ${ }^{1,9}$ Lower frequency ultrasound exhibits low attenuation, and thereby low loss of signal amplitude at deep targets inside the body. A low frequency, however, also means longer wavelength, and thereby larger and less well-defined focal zones. This consequently leads to lower treatment accuracy and higher risk of inflicting damage to tissue outside the specific target. ${ }^{9}$ 
HIFU systems operating at higher frequencies for lower penetration depths have been made widely commercially available for various non-medical aesthetic treatments, typically skin rejuvenation and facial wrinkle reduction. The systems operate at $4 \mathrm{MHz}$ to $10 \mathrm{MHz}$, where focal zones are small enough to be confined to the lower dermis and subcutaneous layers located approximately 2 to $6 \mathrm{~mm}$ below the skin surface. ${ }^{10,11}$ The dimensions of the focal zones generated by such devices are, however, fundamentally close to, and most often larger than, the total thickness of the human dermis. ${ }^{12}$ More superficial therapeutic targets, eg, tumors localized in the epidermis, basement membrane and upper dermis 0.5 to $1.5 \mathrm{~mm}$ from the skin surface, are thus out of scope for all such devices.

Recently, it has been demonstrated in preclinical and clinical studies that HIFU operating at $20 \mathrm{MHz}$ produces very small and well-controlled focal zones at close focal distances. ${ }^{13}$ This can be used to safely target features near the surface of the skin, and reproducibly deliver a clinically efficient thermal and mechanical insult that will kill cells. Highfrequency HIFU therefore becomes relevant to a very large range of dermatological and aesthetic indications and conditions. ${ }^{14-17}$ The principle of HIFU used for dermatological indications is illustrated in Figure 1.

In the very broad spectrum of dermatological diseases and conditions relevant for high-frequency HIFU, premalignant lesions and skin cancers are of major interest due to their immediate clinical relevance. However, a range of other common benign conditions can also lead to both medical and socio-psychological problems, and therefore require resources in both the private and public health sectors. In this latter group, Seborrheic Keratosis (SK) is a major contributor, as it is extremely common and usually multiple. ${ }^{18-20}$

SK is a benign epidermal lesion that can appear on any part of the body except for the mucous membranes, palms, and soles. Most common locations are on the chest, front- and back-trunk, but can also involve the scalp, face, neck, and extremities. ${ }^{20} \mathrm{SK}$ typically presents as oval, slightly raised, light-brown to black, sharply demarcated papules or plaques. Each field rarely grow to more than $3 \mathrm{~cm}$ in diameter, but may be present in very high quantities, densely covering entire body areas. If left untreated, they can grow to thicker warty lesions that can appear crumbly on the surface, but have a raw moist base if the surface is removed. ${ }^{18-23}$

Given the benign and asymptomatic nature of SK, treatment is not generally prescribed for medical reasons. Removal of lesions may nevertheless be performed to eliminate suspicion of developing malignancies. ${ }^{23}$ Other typical reasons for therapy include persistent irritation from, eg, clothing, which, as a secondary medical effect, leads to

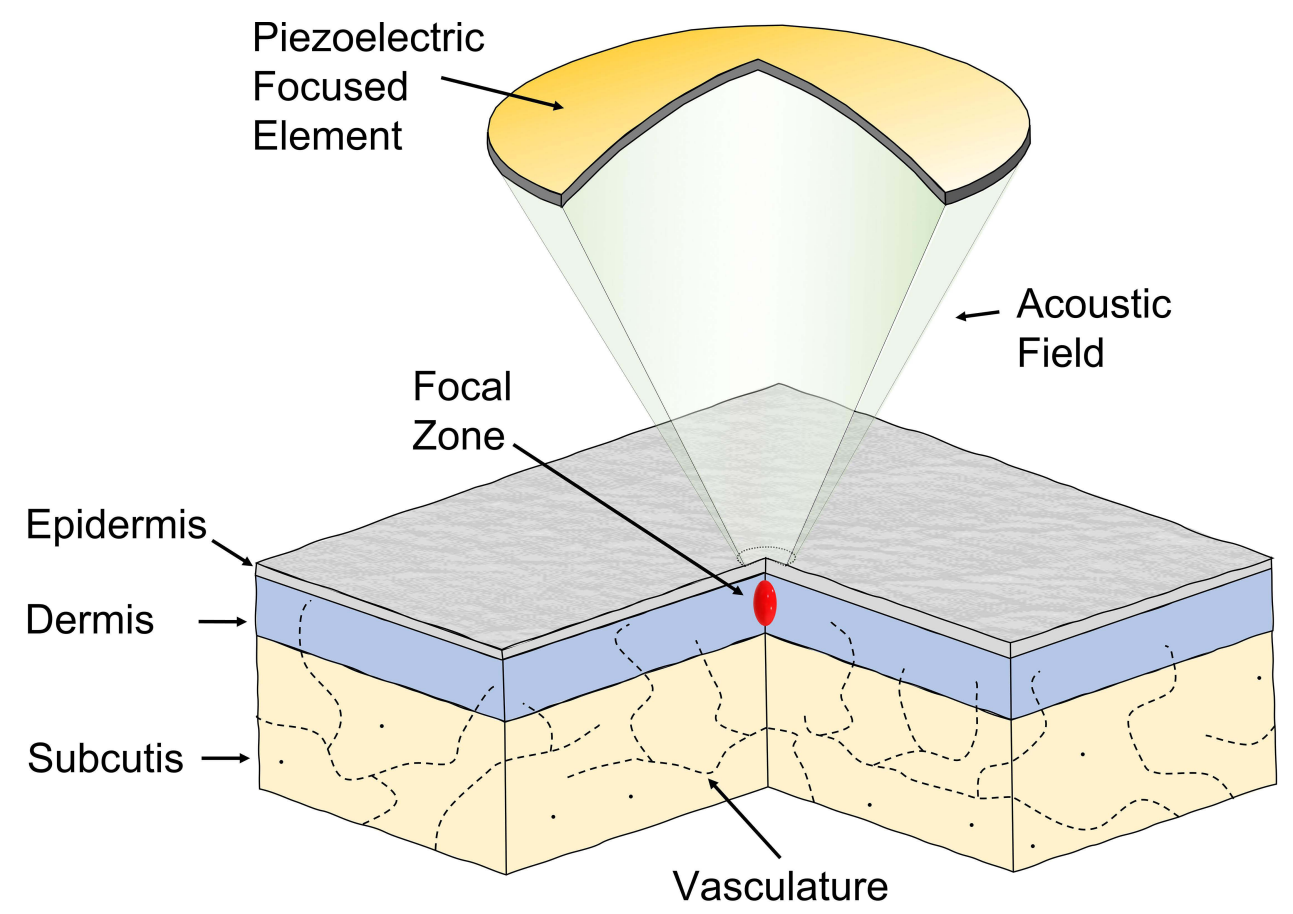

Figure I High-frequency HIFU applied to the human skin. ${ }^{13}$ At $20 \mathrm{MHz}$, the focal zone is sufficiently small to prevent damages to the deeper part of the dermis and subcutaneous layer below. 

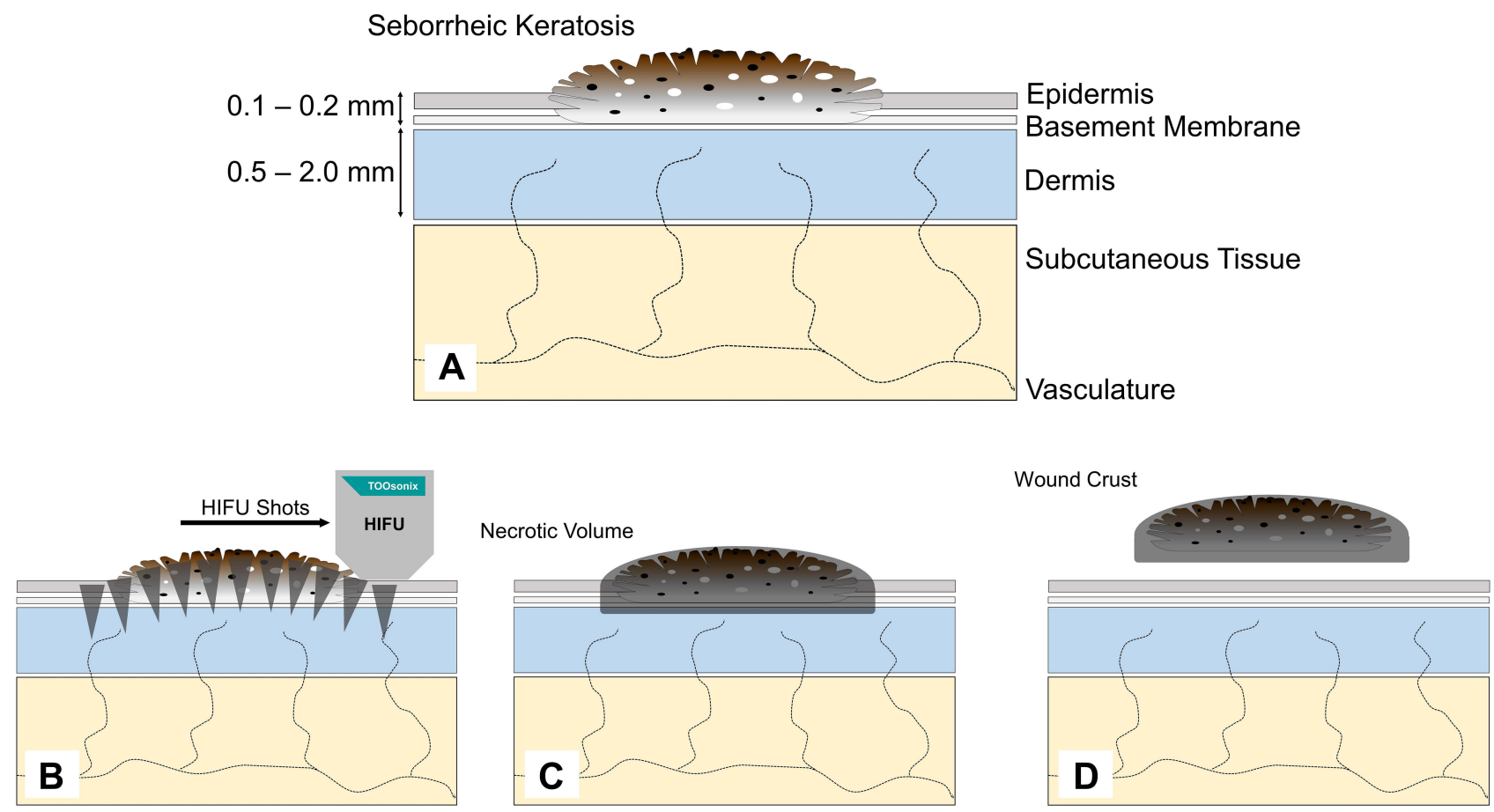

Figure 2 Dynamics in removal of seborrheic keratosis by high-frequency HIFU. (A) An epidermal seborrheic keratosis (SK) is located in the skin. (B) Shoulder-by-shoulder HIFU doses are placed to cover the SK-field including a small margin. (C) A necrotic volume containing SK cells is formed directly after treatment. (D) The wound crust is spontaneously released after I-2 weeks after damaged cells have been replaced.

inflammation, ulceration, bleeding and/or infections. ${ }^{24,25}$ The vast majority of removal procedures, and especially in patients with multiple lesions, are, however, performed in private dermatology clinics for purely cosmetic reasons. The motivation for removal is typically embarrassment from the stigmatizing appearance of the lesion and a desire to look younger. $^{25}$

Inspired by the above-mentioned efficacy of HIFU used both for deep internal cancers and dermatological treatments, high-frequency HIFU used on SK is therefore proposed as a straightforward option for further study.

Used as an ablative method, the $20 \mathrm{MHz}$ HIFU by intention produces a superficial necrotic volume that will form a thin external wound crust that heals over the following few weeks. Scar sequelae have been very limited in previous superficial treatments, and only sporadic cases with further fibrous change of an already damaged dermis have been observed. ${ }^{14}$ The $20 \mathrm{MHz}$ HIFU method has therefore been concluded to be both safe and efficient with respect to production of controlled skin ablations. ${ }^{14-17}$ The proposed process and dynamics of removal of SK by HIFU are illustrated in Figure 2.

This study presents the first use of the novel $20 \mathrm{MHz}$ HIFU method applied to Seborrheic Keratosis in human skin. No indication-specific guidance and no past study were available from the medical literature. Clinical material is presented, supplemented with illustrative case reports.

\section{Patients and Methods}

\section{Subject Recruitment}

The study was open-label and integrated in the ongoing treatments practiced in the clinic. HIFU treatment was offered as an optional alternative to cryosurgery or surgical methods to patients with SK. This included subjects, who, for different personal reasons or preferences, abstained from the routine therapy, eg, due to earlier experience. Thus, fixed standards of inclusion- and exclusion criteria were not used.

The material presented in the study is consecutive and includes all HIFU-treated SK subjects, who visited the clinic within the period from September 2020 to June 2021 in accordance with the recommendations for follow-up. 
Ethics committee approval was not necessary prior to this study, as treatments were performed on a CE marked medical device within the scope of its intended purpose (on-label), using standard settings as described in the IFU for the device only, and generally adhering to normal clinical practice. Patients were informed before treatments, and gave their consent to participation in the study and publication of anonymized photos. The principles of the Helsinki Declaration II were followed.

\section{Pre-Treatment Assessment}

Clinical dermoscopy evaluation of each SK lesion/field was performed using a Fotofinder Medicam 1000 (FotoFinder Systems GmbH, Bad Birnbach, Germany). Included lesions were clinically observed in the dermoscope presenting the typical flat, slightly raised, or pedunculated papules with one or more of the following additional characteristics: homogeneous hyperpigmented area, curved lines, network-like structures, brown and white clods and looped vessels.

\section{HIFU Treatment}

Treatments were performed using a TOOsonix System ONE-M (TOOsonix A/S, Hoersholm Denmark) operating at a frequency of $20 \mathrm{MHz} \pm 5 \%$. The system is shown in Figure 3.

A handpiece with Nominal Focal Depth (NFD) of $0.8 \mathrm{~mm}$ was used in all treatment cases, thus utilizing the most superficial focal depth available from the standard range delivered with the system. Focal zones with localized heating to approximately $60-65^{\circ} \mathrm{C}$ could thereby be positioned and confined to the epidermis and upper quarter of the dermis only. ${ }^{13,14}$

"Shoulder-by-shoulder" HIFU doses of 0.99 to $1.2 \mathrm{~J} /$ dose at durations of $150 \mathrm{~ms} /$ dose were administered with a distance of 1 to $2 \mathrm{~mm}$ between each dose to cover an entire lesion field with a small circumferential margin of approximately $1 \mathrm{~mm}$. Repetition of each dose was approximately 1-2 seconds. Progress and status of treatments were

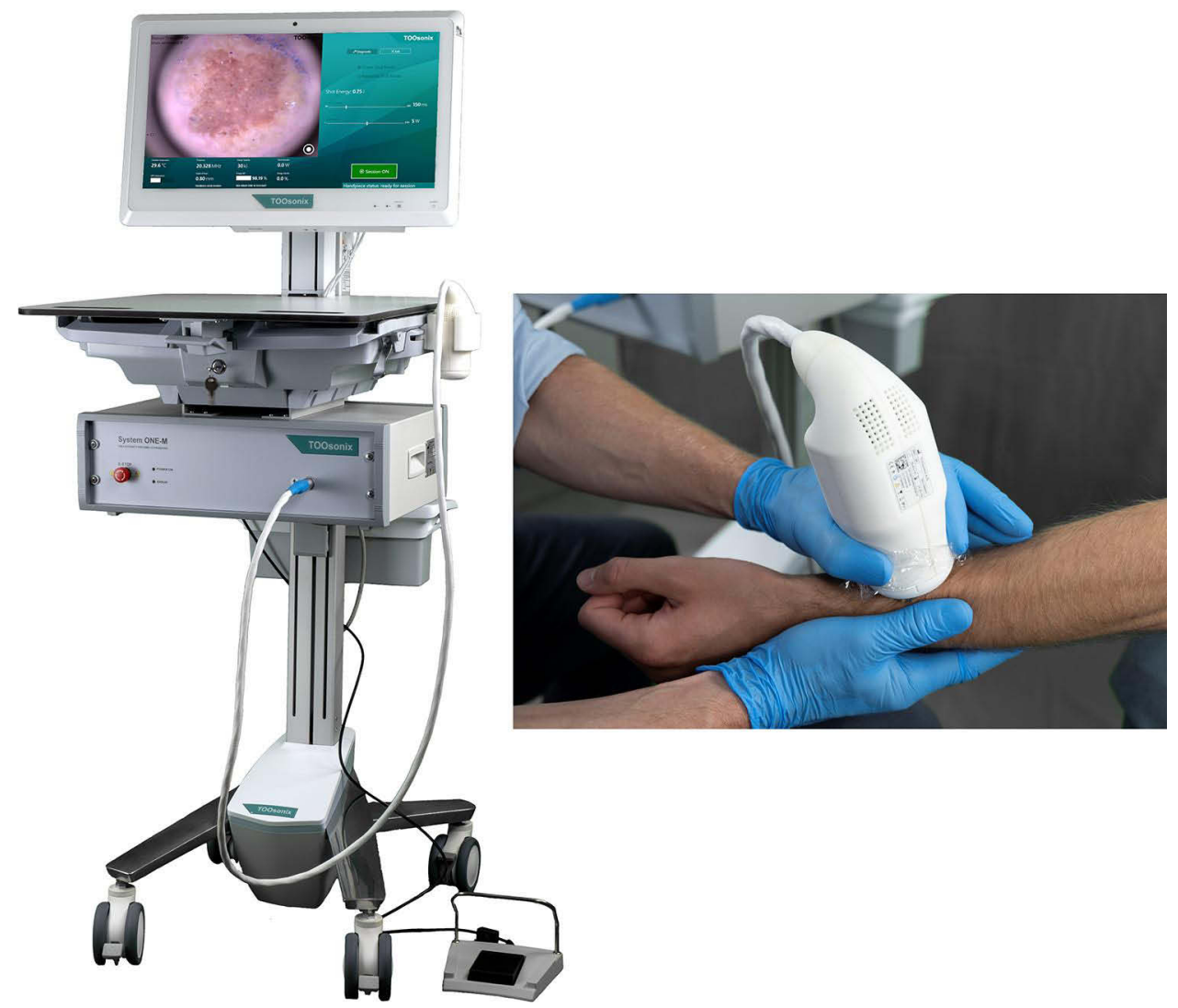

Figure 3 TOOsonix HIFU system operating at $20 \mathrm{MHz}$. The handpieces have an integrated real-time video camera allowing accurate control and monitoring of the treatment. 
monitored in real-time via an integrated high resolution digital video camera in the handpiece, that clearly showed treated areas as raised lighter points, where the epidermis was lifted or partly separated. Parker Aquasonic 100 ultrasound gel (Parker Laboratories Inc, NJ, USA) was used to provide acoustic coupling between the handpiece and skin.

Based on previous experience, no pre-treatment topical anesthesia or local intravascular was used prior to treatment.

Treatments were done with one session only, except for two cases where the initial treatment gave partial or no result. In both these cases, a second repeated treatment was administered 4-6 weeks after the initial session.

\section{Statistical Analysis}

For the purpose of the study, the continuous data were characterized by descriptive statistics such as mean and standard deviation. Nominal data are presented as counts of occurrences with percentages. The median of the responses was used as the final result of the study. One-sample Wilcoxon sign rank test was used to assess the difference between the overall response to the reference response: 2 (partial response) and 3 (stable disease). All tests were considered statistically significant at a p-value $<0.005$. The analysis was performed with Minitab v.18 (Minitab Ltd, Coventry, UK).

\section{Post Treatment Clinical Assessment and Rating Scales}

Immediate reactions were noted and documented by dermoscope and macro-photos where relevant. Regular follow-up during the healing phase was conducted, either at physical control visits or e-consultation. The follow-up period in the study ranged from 13 to 107 days with an average of $45.4 \pm 31.1$ (mean $\pm \mathrm{SD}$ ) days depending on the availability and preferences of patients.

Two dermoscopy-trained oncologists performed clinical evaluation and dermoscopy to agree on a rating of overall treatment efficacy. In debatable cases, the final assessment was a jointly agreed compromise between the investigators. Efficacy was rated on a scale from 1 to 4 (1: Complete Response; 2: Partial Response (regression on more than 50\% of the lesion area), 3: Stable Condition, 4: Progressive Condition). In cases where treatments were repeated, the result of the first session was used as the result for statistical analysis.

A similar approach was used to classify the presence of one or more side-effects within the following three categories: Vessels, Scaring and/or Lichen Planus-like Keratosis.

\section{Results}

A total of 11 patients all with Fitzpatrick Type I and Type II skin type were included. The age was between 39 and 67 with an average of 51.5 years and a standard deviation of 13.2. The gender distribution was 8 female (73\%) and 3 male (27\%).

In total, 54 SK were treated. Most subjects had SK in multiple locations, and HIFU was applied in up to 19 lesions per subject. Lesions were localized in different anatomical locations on the body with $52 \%$ located on the front and back trunk, $33 \%$ on the face and neck, and $15 \%$ located on the arms and legs. This anatomical distribution of lesions reflects the normal reported cases from literature. ${ }^{20-24}$ Of the 54 treated SK, 3 (5.6\%) were pedunculated and 51 (94.4\%) were sessile.

The anatomical location and distribution of lesions on each subject is illustrated in Figure 4.

\section{Immediate Effects After Treatment}

In general, HIFU-treated SK showed an immediate circular whitening-effect approximately $2 \mathrm{~mm}$ around each dose target. This was assigned to the denaturing proteins in the affected cells, and a partial release of the epidermal layer, as a result of the rapid local temperature increase. After treatment of larger areas, a mild erythema appeared in the area around the treatment field due to an urticarial histamine release. This reaction gradually decreased over the following 1-2 hours, and did not cause notable pain or discomfort for the subject. Even if the basement membrane was broken during use of the very shallow focal depth of the selected handpiece, no bleeding was observed, and the treated area remained dry and intact. Subjects had no down-time after treatment.

Typical dermoscope pictures of SK before and directly after HIFU treatment are shown in Figure 5. 


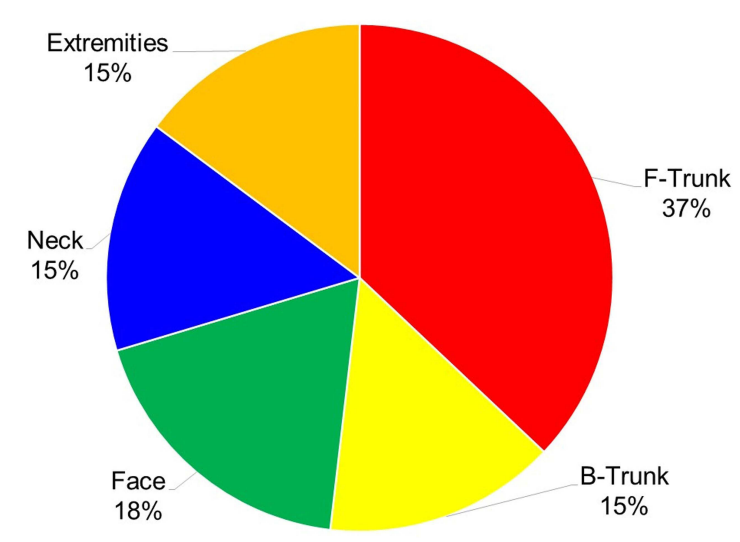

A

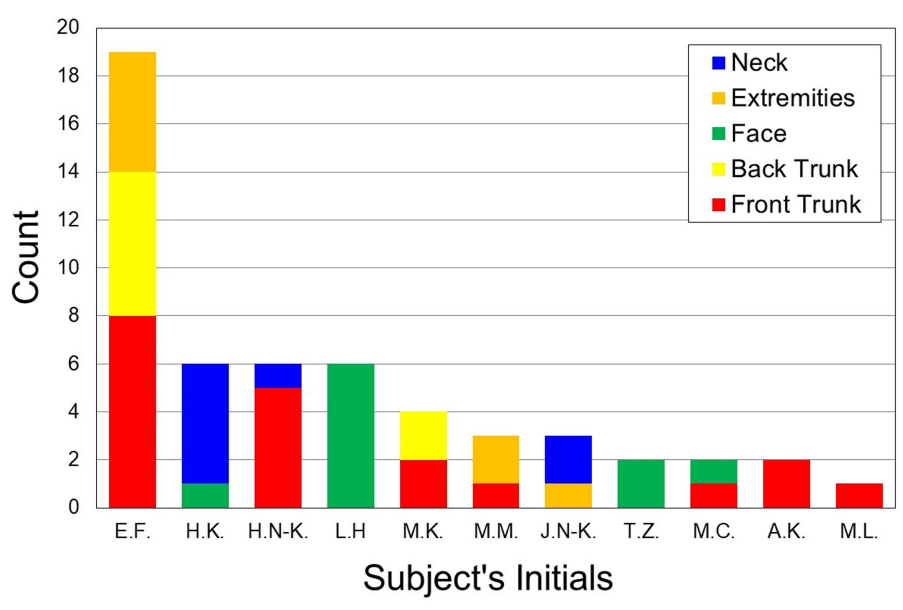

B

Figure 4 (A) Overview of anatomical location of lesions selected for HIFU treatment. (B) Anatomical location for each subject in the study.
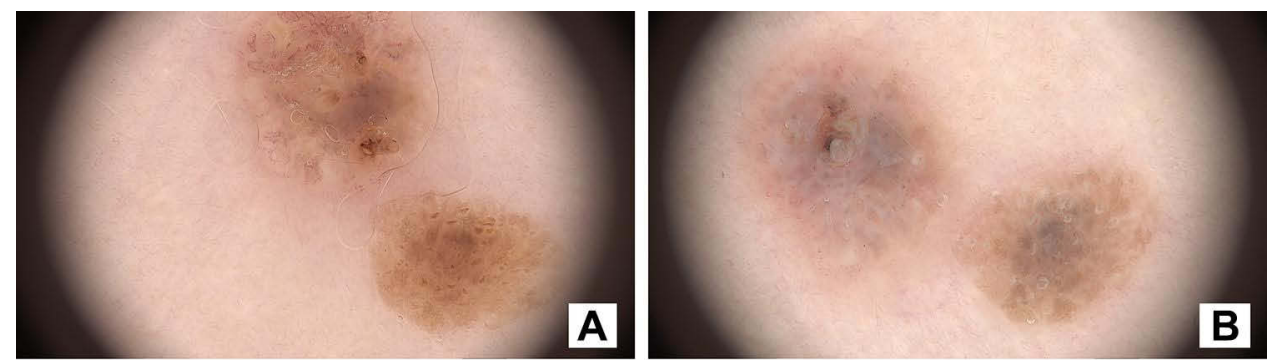

Figure 5 Typical dermoscope appearance of seborrheic keratosis. (A) Before HIFU treatment. (B) Directly after treatment. Whitening of the epidermis and denaturation of the superficial skin structure as a reaction to the thermal and mechanical effects of HIFU can be observed.

\section{Pain from Treatment}

The pain level during HIFU treatment was acceptable to all subjects. There was no single case, when the procedure would have to be stopped due to pain sensations. Immediately after each treatment, patients were asked to score their experience of pain during the treatment on a 0-10-point VAS pain severity scale (Visual Analog Scale). Most subjects scored the pain level to approximately 2, with a maximum score for severity of the pain of 4 .

The pain occurred only temporarily, during exposure to ultrasound, and did not persist after the procedure. Subjects compared the sensations to "pin pricks".

\section{Healing Phase After Treatment}

The description of the dynamics of healing was based on direct verbal reports given by the subjects. In most cases, subjects reported that 7-14 days after the procedure, they observed the exfoliation/separation of a thin wound crust, and the appearance of a pink rebuilt epidermis. None of the subjects reported any complaints about pain or other discomforts in the treated area during the healing period.

\section{Efficacy of Treatment}

Of the 54 SK removed with HIFU, 52 cases (96.3\%) showed a positive response to treatment with 37 (68.5\%) cases classified as Complete Response and 15 (27.8\%) cases with Partial Response. Two cases (3.7\%) did not respond to treatment and were classified a Stable Condition. No subjects experienced worsening of their condition, and no treatment therefore received the classification of Progressive Condition. 
Table I Overview of Subjects and Results of HIFU Treatments (Full Data are Available as Additional Information from the Journal Internet Page)

\begin{tabular}{|c|c|c|c|c|c|c|c|}
\hline \multirow[t]{2}{*}{ Subject } & \multirow[t]{2}{*}{ No. of Lesions } & \multirow[t]{2}{*}{ Gender } & \multirow[t]{2}{*}{ Age } & \multicolumn{4}{|c|}{ Outcome at Final Follow-Up } \\
\hline & & & & Complete Response & Partial Response & Stable Condition & Progressive Condition \\
\hline E.F. & 19 & Female & 61 & 16 & 3 & 0 & 0 \\
\hline L.H & 6 & Male & 61 & 3 & 2 & 1 & 0 \\
\hline H.K. & 6 & Female & 58 & 3 & 3 & 0 & 0 \\
\hline H.N-K. & 6 & Female & 31 & 6 & 0 & 0 & 0 \\
\hline M.K. & 4 & Female & 47 & 1 & 3 & 0 & 0 \\
\hline M.M. & 3 & Male & 60 & 1 & 2 & 0 & 0 \\
\hline J.N-K. & 3 & Female & 66 & 2 & 1 & 0 & 0 \\
\hline A.K. & 2 & Female & 39 & 2 & 0 & 0 & 0 \\
\hline M.C. & 2 & Female & 67 & 0 & 1 & 1 & 0 \\
\hline T.Z. & 2 & Male & 44 & 2 & 0 & 0 & 0 \\
\hline M.L. & 1 & Female & 33 & 1 & 0 & 0 & 0 \\
\hline Total & 54 & & & 37 & 15 & 2 & 0 \\
\hline
\end{tabular}

In one case, where only Partial Response was observed (illustrated as Case 1 below), the SK was very large, thick and with a porous warty texture. Full removal was therefore not expected in a single session. A second session with repeat treatment was therefore administered. Likewise, repeat treatments to other lesions classified as Partial Response were administered with a second repeat treatment to further improve the result. Only results of the initial treatment were used for statistical analysis. The summary of the treatment is shown in Table 1.

\section{Clinical Result}

The presented data confirm the clinical benefit of SK treatment using HIFU by a clinical response rating ranging from 1 to 3 (CR to $\mathrm{SR}$ ) with average score of $1.4 \pm 0.6$ (mean $\pm \mathrm{SD}$ ) points and a median equal to 1 . The statistical significance of the final score difference from reference scores of 3 (Stable Disease) and 2 (Partial Response) has been confirmed by the one sample Wilcoxon sign rank test with p-value $<0.005$.

\section{Side Effects and Safety Profile}

Out of 54 treated lesions in total, 18 (33.4\%) did not lead to any observed side effects. The most commonly clinically observed side effect of therapy was mild local skin erythema. In dermoscopic examination, this corresponded to 26 observations (48.1\%) of superficial telangiectasia and capillary blood network in the direct treatment area.

Scarring was observed by dermoscopic imaging in 6 lesions (11.1\%). Scarring was mild and not related to bacterial infections, etc. It is not known if this scarring and fibrotic tissue changes were due to the HIFU treatment or pre-existing changes in the dermis originating from the SK lesion.

In two cases with treatment of large lesions (3.7\%), brownish-gray granularities resembling the structure characteristic of Lichen Planus-like keratosis (LPLK) was observed by dermoscopy. These are variants of SK or solar lentigo in immune regression, ${ }^{22}$ and thus signs of on-going slower healing. These effects could thus gradually involute over time, or potentially be removed by subsequent repeat treatment after stabilization.

In other two cases $(3.7 \%)$ vessels together with mild scarring were observed as well.

All side effects were within expectations and deemed as mild by the subjects. No adverse event or serious adverse events were observed. 


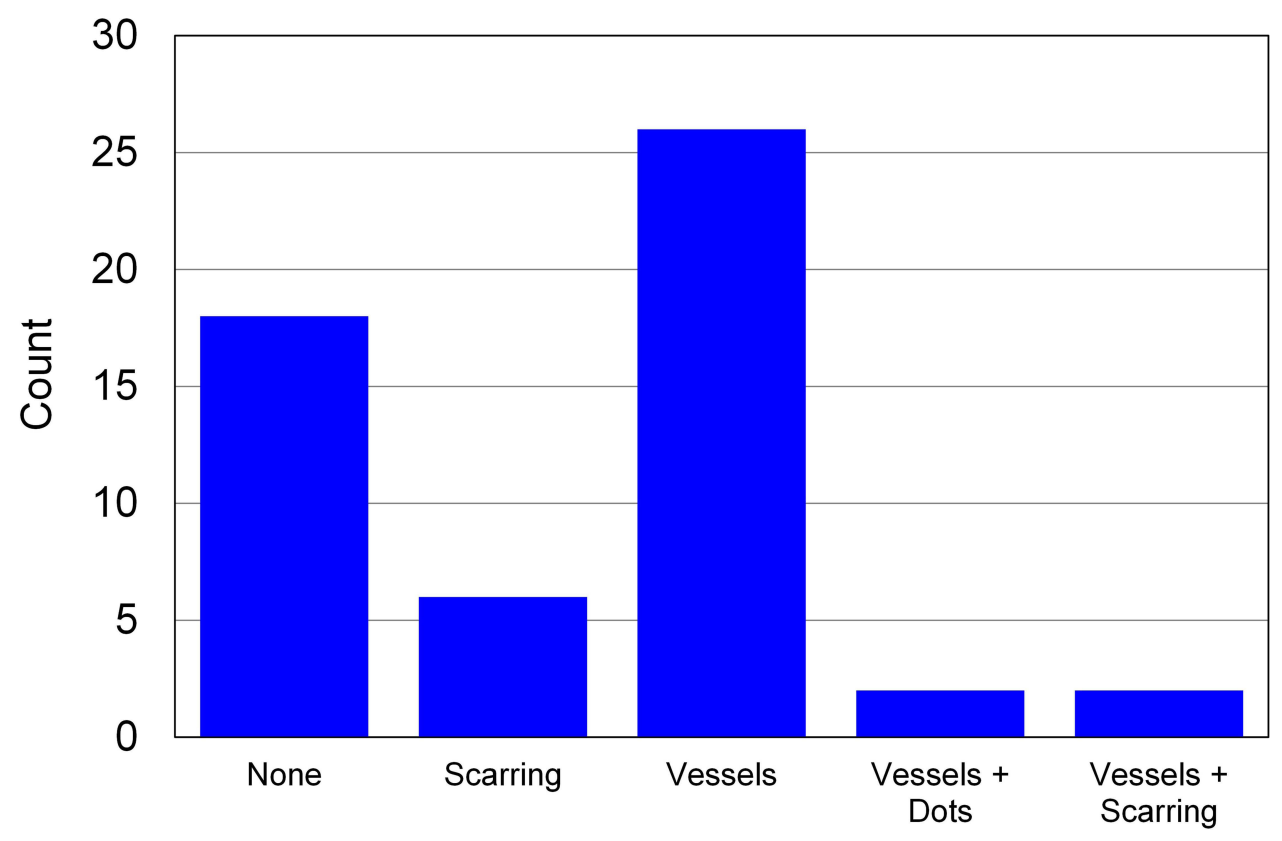

Figure 6 Summary chart of dermoscopic features detected at follow-up.

The dermoscopic observations of the treated areas are summarized in Figure 6.

\section{Treatment Results Illustrated by Selected Case Reports Case Report I}

The subject (M.K) was a 47-year-old female reporting to the clinic concerned about an approximately $6 \mathrm{~cm}$ long ovalshaped convex dark brown lesion with a rough surface on her back, which had grown significantly over the past few months (see Figure 7). In a dermoscopic study, the lesion presented typical features of SK: sharp demarcation, brown clods and coiled vessels. Further whole-body dermoscopic examination revealed several smaller, brighter and flat areas of SK. The described main lesion and three other lesions were subjected to HIFU therapy.

A partial response in the main lesion was observed at the first control visit, 42 days after treatment, but with a clearly positive aesthetic effect for the subject. In the dermoscopic examination, structures of SK: brown papules, loop-shaped vessels and glomeruli were still present in the left side of the lesion. Dermoscopic examination of the right side of the lesion revealed some remaining flat brown clods and grayish-brown granularities resembling LPLK, but with significantly less severity than baseline. Due to this partial response only, HIFU treatment was repeated with normal dosing covering the entire surface of the lesion.

A second control visit took place 28 days after the second HIFU treatment (70 days after first HIFU treatment). Dermoscopic examination still revealed some remaining residues of SK, but with further reduction in the number and size of brown clods and granularities resembling LPLK. Mild telangiectasia was also visible in the dermoscope, but is expected to reduce over the following months of healing.

The final classification of the result after two treatments was still Partial Response, but with a very clear aesthetic improvement for the subject without significant scar-formation, which would otherwise have resulted from, eg, laser or surgical removal. A third treatment may be administered to further reduce the SK after a full healing period of 4-6 months.

\section{Case Report 2}

The subject (M.C) was a 67-year-old woman presenting a larger $(\sim 5 \mathrm{~cm})$ flat lesion on her left breast (Figure 8). Dermoscopic diagnosis indicated a homogeneous area with brown curved lines, clods and looped blood vessels that are typical of SK. Further whole-body dermoscopic examination only revealed one other SK of relevance for removal due to cosmetic reasons. The described main- and secondary lesions were subjected to HIFU therapy. 

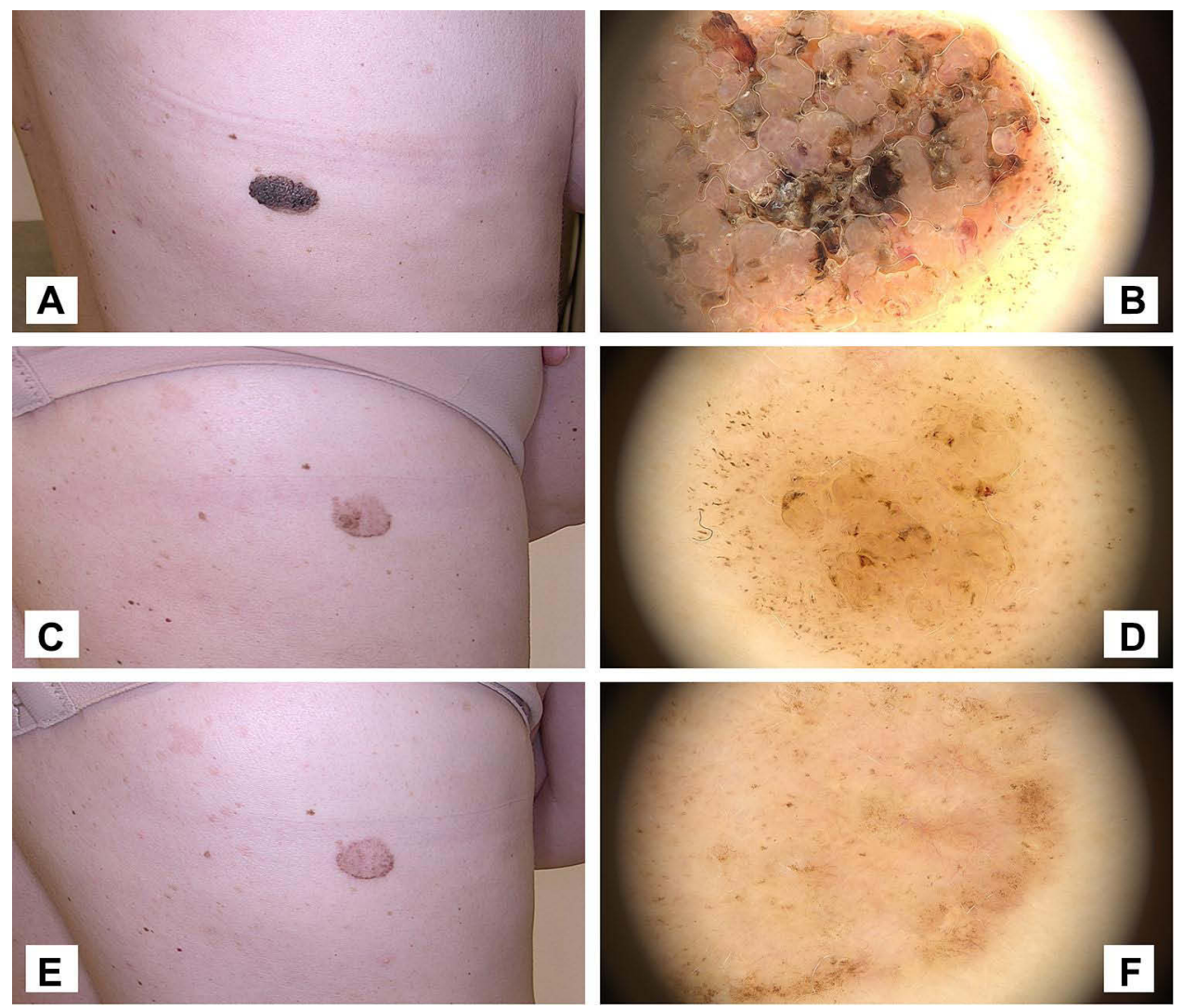

Figure 7 Case I. (A and B) Macro-photo and dermoscope pictures of a large and thick seborrheic keratosis before HIFU. (C and D) Macro-photo and dermoscope pictures 6 weeks after initial HIFU treatment. The seborrheic keratosis has clearly reduced in thickness and the majority of brown clods and coiled vessels have been removed. The lesion is however still clearly visible, in particular in the periphery of the original lesion. A second repeat treatment was administered. (E and $\mathbf{F})$ Macro-photo and dermoscope picture at follow-up visit 10 weeks after first treatment (4 weeks after second treatment). The visual appearance is significantly improved, but seborrheic keratosis is still visible, and a third treatment will be needed to fully remove the lesion.
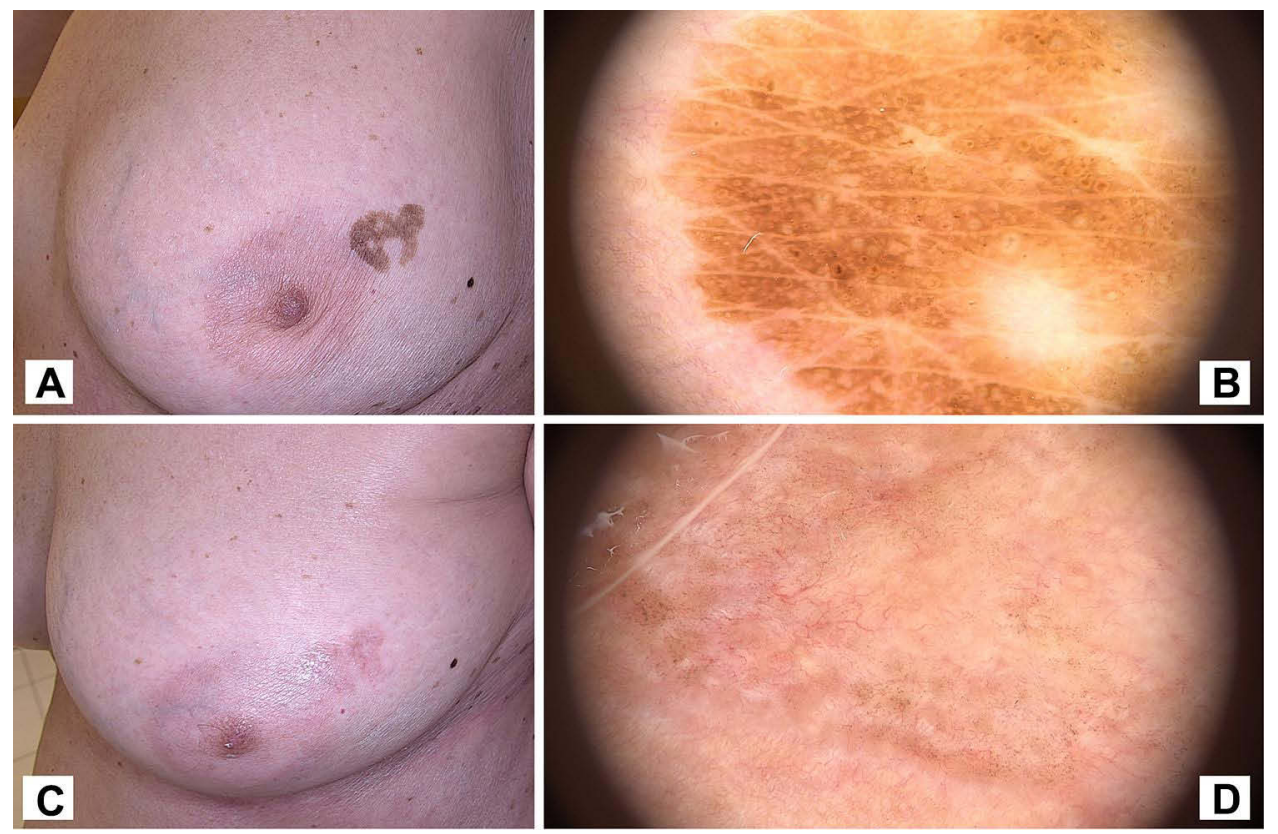

Figure 8 Case 2. (A and B) Macro-photo and dermoscope picture of large seborrheic keratosis on left breast before HIFU. (C and D) Macro-photo and dermoscope picture of treated area at control visit 4 weeks after HIFU treatment. 


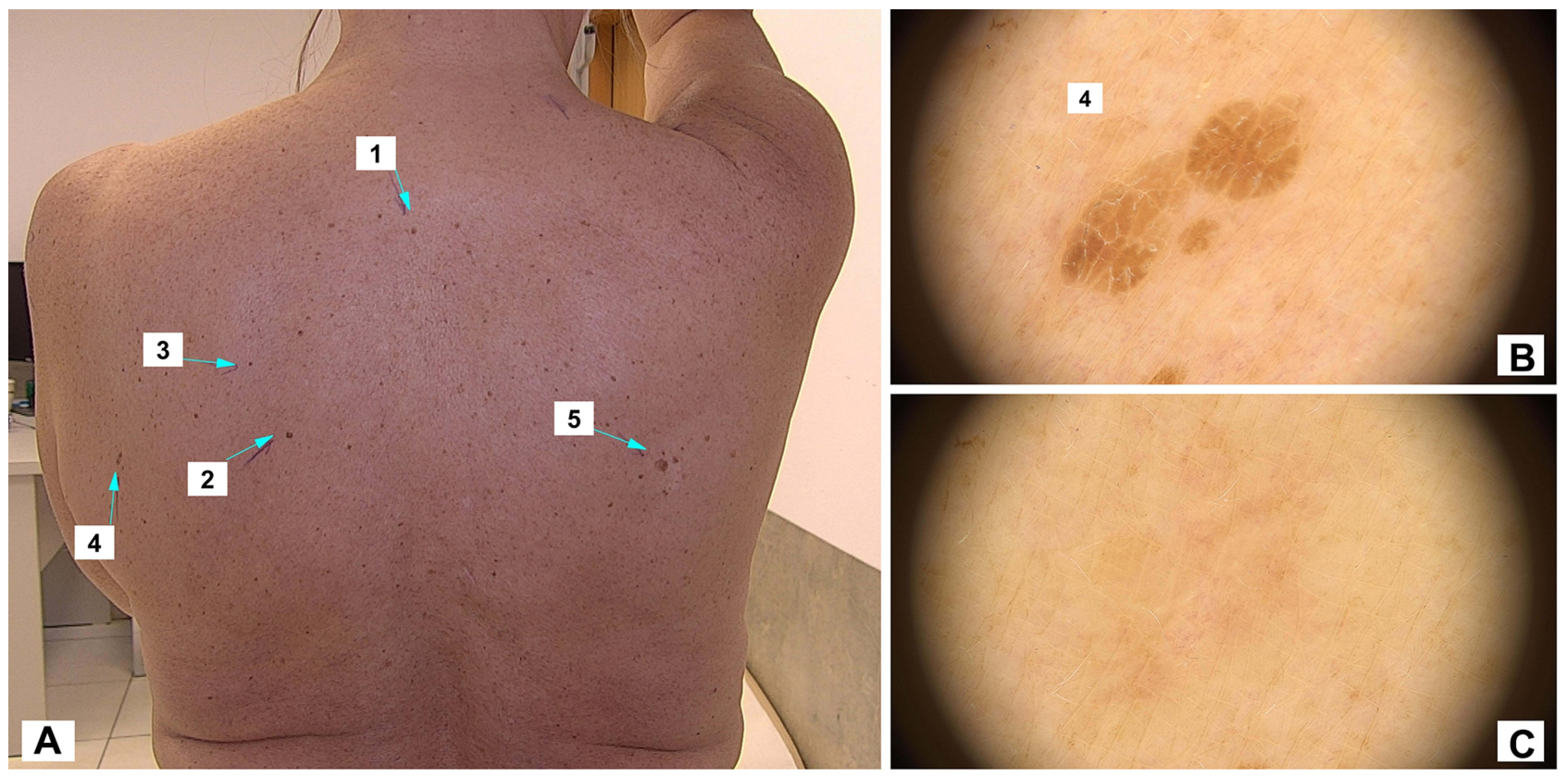

Figure 9 Case 3. (A) Macro-photo of 5 selected seborrheic keratoses lesions for HIFU treatment. (B) Dermoscope picture of lesion number 4 before HIFU. (C) Dermoscope picture of lesion number 4 at the control visit 5 weeks after treatment.

Treatment was performed in a single session, with follow-up after 4 weeks. At follow-up, some inflammatory redness was still visible, and a small network of telangiectasias were visible by dermoscopic examination. Likewise, some signs of brown pigment could still be observed.

The treatment result was thus classified as a Partial Response. However, clinically the aesthetic result was seen as positive from both doctor and subject with a clear clinical and visual improvement. A second repeat treatment after some additional 4-6 months healing is likely to give a complete response.

\section{Case Report 3}

The subject (E.F) was a 61-year-old woman with multiple small $(2-5 \mathrm{~mm})$ flat lesions on the back. In dermoscopic examination, they all showed the normal characteristics of SK with brown curved lines with additional brown clods and looped vessels in the larger lesions. Macroscopic as well as dermoscopic pictures of the lesions are given in Figure 9.

A total of 6 lesions were selected for HIFU treatment in a single session. At follow-up after 5 weeks, inflammatory redness, telangiectasia and brown spots were almost not visible, and it was clinically not possible to distinguish the target areas from the normal surrounding skin. The classification of the treatments was therefore Complete Response for all treated lesions.

\section{Discussion}

Seborrheic Keratosis is, besides birthmarks, the most common benign growth of epidermal cells. Most people develop at least one SK in their lifetime, usually forming de novo in adulthood. Many elderly people will have multiple lesions, sometimes counted in hundreds, covering entire body parts. ${ }^{18,19}$

SK is caused by many factors, among which the most important are skin aging, UV exposure, genetic predisposition, and chronic mechanical irritation of the skin. The typical onset of SK is generally in the fourth to fifth decade of life, with a peak around the age of $60 .{ }^{18-23}$ The incidence rate of SK is therefore expected to follow the general upwards age-shift in the population in the industrialized world over the next decades.

Smaller protruding SK are typically removed by curettage, while cryosurgery is recommended for flat SK. A combination of the two methods is also reported, ie, with an initial freezing of the SK followed immediately by curettage. For larger areas or more complex SK, surgical excision, ablative erbium, YAG or $\mathrm{CO}_{2}$ lasers can be used, but 
these methods carry high risks of bacterial infection, scar-formation and pigmentary changes. Topical and systemic therapies are in their early stages, but have so far not proved to be a viable option for efficient treatment. ${ }^{24-27}$

As it is often the case with dermatological therapies, there is no single general preference among the above available therapeutic options. Incomplete treatment results with reoccurrences, scarring or pigment changes are not uncommon. Similarly, the very large variation in the type of SK observed in patients requires several different methods to be applied for optimal results. New treatment modalities, which can cover a wider field of both small and large areas, be used on both thick and thin SK, and are feasible to operate for private clinics, are therefore needed.

The current study presents the first results of a new high-frequency HIFU modality for removal of Seborrheic Keratosis in an initial study involving 11 subjects with a total of 54 treated lesions.

Treatments were performed by covering the target area with HIFU doses, typically using less than one minute per SK lesion. The system provides real-time monitoring of the treatment area, and practitioners could therefore monitor and secure accurate targeting and correct skin response during the treatment.

Pain was typically scored at $2-4$ on a $0-10$ point VAS scale, and was reported to be present only during the HIFU dosing. The treatment is therefore comparable in time and pain level to cryotherapy, but significantly less painful than laser-treatment and less intrusive than curettage. In case treatment is performed on specially pain-sensitive anatomic locations or sensitive patient groups, use of topical anesthesia (EMLA ${ }^{\circledR}$, AstraZeneca) has previously been shown to decrease treatment pain significantly without affecting treatment results. ${ }^{16}$ If necessary, topical anesthesia can therefore be prescribed as a standard self-administered pre-treatment option.

The study resulted in improvement in $96 \%$ of the cases after a single HIFU session. $68 \%$ had a complete removal, while $29 \%$ had more than $50 \%$ reduction in the condition compared to baseline. Within the latter group having partial reduction only, some SKs were very thick and crusty, and were therefore not expected to be removable with one HIFU session only. These lesions could however be further reduced in a second treatment, and potentially fully removed in subsequent 3rd and 4th treatments. While this is more time-demanding than eg single surgical procedures or laser ablation, it is a more gentle process, that does not carry the same high risks of pain, infections, scarring and depigmentation.

Only $4 \%$ of the 54 treated lesions did not respond to treatment. As reported in other cases using $20 \mathrm{MHz} \mathrm{HIFU}$, it is, however, expected that this could be improved by using other settings and/or a slightly deeper focal depth.

Overall, the HIFU treatment was beneficial to all subjects in the study, producing an average response score of 1.35 on a 1-4 point efficacy-scale. Statistically, this was found to be significantly different in comparison to the reference levels of 2 and 3 using a one-sample Wilcoxon sign rank test.

Side effects were generally mild, with a temporary urticarial redness around the treatment field during the first few hours after treatment, and inflammatory redness in the treatment area during healing. Mild scarring was observed in $11 \%$ of the cases, but it is not known to what extent this was caused by the HIFU or pre-existing tissue changes created during the growth of the SK. No adverse events were noted during the study.

\section{Conclusion}

$20 \mathrm{MHz}$ HIFU is considered a new and very broadly applicable treatment modality for SK. In this study, a very good safety profile and high efficacy has been demonstrated. A limiting factor of this initial study is, however, the relatively small number of subjects. Further studies, eg, with more participants, blinded scoring and/or randomized comparisons with comparative methods would be beneficial to further characterize the safety and efficacy of the method in a broader population.

\section{Statement of Ethics}

This study was conducted ethically and in accordance with the World Medical Association Declaration of Helsinki. The subjects received information about the study and accepted to participation in treatment and publication of anonymized photos and data from their treatments.

\section{Funding}

There were no funding sources for this work. 


\section{Disclosure}

The HIFU device was provided by TOOsonix to the Old Town Clinic for clinical work. Dr Jacek Calik reports nonfinancial support from Toosonix, during the conduct of the study. Dr Tomasz Zawada and Mr Torsten Bove report a patent EP3589367B1 issued to TOOsonix A/S. The authors report no other conflicts of interest in this work.

\section{References}

1. Ellens NPK, Partanen A. Preclinical MRI-guided focused ultrasound: a review of systems and current practices. IEEE Trans Ultrason Ferroelectr Freq Control. 2017;64(1):291-305. doi:10.1109/TUFFC.2016.2609238

2. Guillaumier S, Peters M, Arya M, et al. A multicentre study of 5-year outcomes following focal therapy in treating clinically significant nonmetastatic prostate cancer. Eur Urol. 2018;74:422-429. doi:10.1016/j.eururo.2018.06.006

3. Peek MCL, Wu F. High-intensity focused ultrasound in the treatment of breast tumours. Ecancer. 2018;12:1-10. doi:10.3332/ecancer.2018.794

4. Quinn SD, Gedroyc WM. Thermal ablative treatment of uterine fibroids. Int J Hyperthermia. 2015;31(3):272-279. doi:10.3109/ 02656736.2015 .1010608

5. Lang BH, Wu ALH. High Intensity Focused Ultrasound (HIFU) ablation of benign thyroid nodules - a systematic review. $J$ Ther Ultrasound. 2017;5:1-9. doi:10.1186/s40349-017-0091-1

6. Barile A, Arrigoni F, Zugaro L, et al. Minimally invasive treatments of painful bone lesions: state of the art. Med Oncol. 2017;34(4):53. doi:10.1007/s12032-017-0909-2

7. Lamsam L, Johnson E, Connolly ID, Wintermark M, Gephart MH. A review of potential applications of MR-guided focused ultrasound for targeting brain tumor therapy. Neurosurg Focus. 2018;44(2):1-7. doi:10.3171/2017.11.FOCUS17620

8. Farr N, Wang YN, D'Andrea S, et al. Hyperthermia-enhanced targeted drug delivery using magnetic resonance-guided focused ultrasound: a pre-clinical study in a genetic model of pancreatic cancer. Int J Hyperthermia. 2018;34(3):284-291. doi:10.1080/02656736.2017.1336675

9. Ellens NPK, Hynynen K. Frequency considerations for deep ablation with high-intensity focused ultrasound: a simulation study. Med Phys. 2015;42(8):4896-4910. doi:10.1118/1.4927060

10. Ko EJ, Hong JY, Kwon T-R, et al. Efficacy and safety of non-invasive body tightening with high-intensity focused ultrasound (HIFU). Skin Res Technol. 2017;23:558-562. doi:10.1111/srt.12371

11. Day D. Microfocused ultrasound for facial rejuvenation: current perspectives. Res Rep Focus Ultrasound. 2014;2:13-17.

12. Park JH, Lim SD, Oh SH, Lee JH, Yeo UC. High-intensity focused ultrasound treatment for skin: ex vivo evaluation. Skin Res Technol. 2017;23:384-391. doi:10.1111/srt.12347

13. Bove T, Zawada T, Serup J, Jessen A, Poli M. High-frequency (20-MHz) high-intensity focused ultrasound (HIFU) system for dermal intervention: preclinical evaluation in skin equivalents. Skin Res Technol. 2019;25(2):217-228. doi:10.1111/srt.12661

14. Soegaard S, Aarup V, Serup J, et al. High frequency (20 MHz) High Intensity Focused Ultrasound (HIFU) system for dermal intervention: a 12 Week Local Tolerance Study in minipigs. Skin Res Technol. 2020;26(2):241-254. doi:10.1111/srt.12786

15. Serup J, Bove T, Zawada T, Jessen A, Poli M. High frequency $(20 \mathrm{MHz})$ high-intensity focused ultrasound (HIFU): treatment of actinic keratosis, basal cell carcinoma and Kaposi sarcoma. Skin Res Technol. 2020;26:824-831. doi:10.1111/srt.12883

16. Serup J, Bove T, Zawada T, Jessen A, Poli M. High-frequency (20 MHz) high-intensity focused ultrasound: new ablative method for colorindependent tattoo removal in 1-3 sessions. An open-label exploratory study. Skin Res Technol. 2020;26:839-850. doi:10.1111/srt.12885

17. Bove T, Zawada T, Jessen A, Poli M, Serup J. Removal of common warts by high-intensity focused ultrasound: an introductory observation. Case Rep Dermatol. 2021;13:340-346. doi:10.1159/000515075

18. James WD, Berger TG, Elston DM, eds. Andrews Diseases of the Skin. 12th ed. Philadelphia: Elsevier; 2016.

19. Elgart GW. Seborrheic keratoses, solar lentigines, and lichenoid keratoses. Dermatoscopic features and correlation to histology and clinical signs. Dermatol Clin. 2001;19(2):347-357. doi:10.1016/S0733-8635(05)70272-2

20. Hafner C, Vogt T. Seborrheic keratosis. J Dtsch Dermatol Ges. 2008;6(8):664-677. doi:10.1111/j.1610-0387.2008.06788.x

21. Mahboob AA, Malvehy J, Braun RP. Atlas of Dermoscopy. 2nd ed. London: Informa Healthcare; 2012.

22. Soyer HP, Argenziano G, Hofmann-Wellenhof R, Johr RH, eds. Color Atlas of Melanocytic Lesions of the Skin. Berlin: Springer; 2007.

23. Minagawa A. Dermoscopy-pathology relationship in seborrheic keratosis. J Dermatol. 2017;44(5):518-524. doi:10.1111/1346-8138.13657

24. Del Roso JQ, Closer A. Look at seborrheic keratoses: patient perspectives, clinical relevance, medical necessity, and implications for management. Clin Aesthet Dermatol. 2017;10(3):16-25.

25. Wollina U. Recent advances in managing and understanding seborrheic keratosis. F1000Research. 2019;8(F1000 Faculty Rev):1520. doi:10.12688/ f1000research.18983.1

26. Sun MD, Halpern AC. Advances in the etiology, detection, and clinical management of seborrheic keratoses. Dermatology. 2021;1-13. doi: $10.1159 / 000517070$

27. Wolina U. Erbium-YAG laser therapy - analysis of more than 1200 treatments. Glob Dermatol. 2016;3(2):268-272. doi:10.15761/GOD.1000171

Clinical, Cosmetic and Investigational Dermatology

Dovepress

\section{Publish your work in this journal}

Clinical, Cosmetic and Investigational Dermatology is an international, peer-reviewed, open access, online journal that focuses on the latest clinical and experimental research in all aspects of skin disease and cosmetic interventions. This journal is indexed on CAS. The manuscript management system is completely online and includes a very quick and fair peer-review system, which is all easy to use. Visit http://www. dovepress.com/testimonials.php to read real quotes from published authors.

Submit your manuscript here: https://www.dovepress.com/clinical-cosmetic-and-investigational-dermatology-journal 